\title{
Atypical recovery from stress induced cardiomyopathy in a patient with anorexia nervosa
}

\author{
Larisa Dzirlo${ }^{1}$, Daniel Kiblboeck ${ }^{2}$, Johann Reisinger ${ }^{3}$, Stefan Eibl $^{4}$, Martin Frömmel ${ }^{4}$ and Monika Graninger ${ }^{1}$ \\ ${ }^{1}$ Third Department of Internal Medicine and Psychosomatics, St. Vincent Hospital, Vienna, Austria \\ ${ }^{2}$ Department of Cardiology and Medical Intensive Care, Med Campus III., Kepler University Hospital, Linz, Austria \\ ${ }^{3}$ Department of Internal Medicine / Cardiology, Hospital of the Sisters of Charity, Linz, Austria \\ ${ }^{4}$ First Department of Internal Medicine (Cardiology), St. Vincent Hospital, Vienna, Austria
}

\begin{abstract}
Anorexia nervosa is a psychiatric disease which is very often complicated by different cardiological abnormalities. There are many case reports, which described stress induced cardiomyopiathy, as a severe cardiological complication. Our unique case shows that during recovery from stress-induced cardiomyopathy, transient apical wall thickening (TAWT) can develop, mimicking apical hypertrophic cardiomyopathy.
\end{abstract}

\section{Case report}

A 39-year-old female was admitted to our hospital because of anorexia nervosa with a body mass index of 10.5 and severe obsessivecompulsive disorder. Due to a spontaneous severe hypoglycaemia of $22 \mathrm{mg} / \mathrm{dl}$ and electrolyte disorders (sodium $128 \mathrm{mmol} / \mathrm{l}$, potassium $2.8 \mathrm{mmol} / \mathrm{l}$, chloride $89 \mathrm{mmol} / \mathrm{l}$ ) she was transferred to the medical intensive care unit. She was disorientated and presented with a slurred speech which recovered after intravenous glucose administration. Her blood tests showed leukopenia (2.9 G/l), thrombocytopenia (91 G/l) with elevated transaminases (AST 1487 U/l, ALT 1425 U/l, GGT 177 $\mathrm{U} / \mathrm{l})$, lactate dehydrogenase (732 U/l) and creatine kinase (1813 U/l), while troponin levels were within normal range. Chest X-ray was normal. An electrocardiogram (ECG) showed sinus bradycardia and T-waves inversion in lead II, III, aVF, and V4-V6. Echocardiography revealed wall motion abnormalities of the apical and midventricular segments, typical for Tako-Tsubo cardiomyopathy and a pre-existing pericardial effusion above the right ventricle. These wall motion abnormalities regressed completely over two weeks. However, enddiastolic apical wall thickness increased to $14 \mathrm{~mm}$. Therefore, she was scheduled for cardiac magnetic resonance imaging which showed thickening of the apex of the left ventricle (apical interventricular septum $19 \mathrm{~mm}$ ) mimicking an apical hypertrophic cardiomyopathy. There was no evidence of myocarditis or myocardial oedema.

Due to enteral feeding via a nasogastric tube and psychological treatment, the patient recovered clinically within 3 months. Laboratory parameters normalised and the patient gained weight to a body mass index of 13. Echocardiography at follow up after 3 months showed a complete regression of the apical myocardial thickening and the pericardial effusion.

Anorexia nervosa is a psychiatric disease which is very often complicated by different cardiological abnormalities such as bradycardia, hypotension, mitral valve prolapse, or arrhythmias due to hypokalaemia or hypomagnesemia [1]. Enormous psychical stress and hypoglycaemia sometimes lead to catecholamine-mediated reactions and development of stress-induced cardiomyopathy [2]. Our unique case shows that during recovery from stress-induced cardiomyopathy, transient apical wall thickening (TAWT) can develop, mimicking apical hypertrophic cardiomyopathy. TAWT which mimics apical hypertrophic cardiomyopathy during recovery from stress-induced cardiomyopathy has been reported, but not in patients with anorexia nervosa [3]. TAWT is one of the less-known phenomena of stressinduced cardiomyopathy. There are two mechanisms that could explain development of TAWT during the course of stress-induced cardiomyopathy. Firstly, TAWT can be a result of inflammation or interstitial oedema, for example in acute myocarditis. In our patient, we had no evidence of inflammation or myocardial oedema. A second theoretical mechanism could be effective via catecholamine stimulation. This may be related to the negative inotropic effect of epinephrine- $\$ 2$ adrenergic receptor- $\mathrm{G}_{\mathrm{I}}$ and the positive inotropic effect of norepinephrine- $\beta 1$ adrenergic receptor- $G_{s}$, which lead to stress-induced cardiomyopathy [4]. Continuous $B$-adrenergic stimulation is known to cause cardiac hypertrophy, and such stimuli may also cause TAWT. One study showed that development of TWAT during the course of stress-induced cardiomyopathy is associated with more cardiac complications and higher hospital mortality [3]. Our case shows that the patients with anorexia nervosa are critically ill patients, and a cardiological examination has to be obligatory.

\section{References}

1. Thurston J, Marks P (1974) Electrocardiographic abnormalities in patients with anorexia nervosa. Br Heart $J$ 36: 719-723. [Crossref]

2. Ono T, Kasaoka S, Fujita M, Yamashita S, Kumagai K, et al. (2009) Complete recovery from severe myocardial dysfunction in a patient with anorexia nervosa. J Cardiol 54 480-484. [Crossref]

Correspondence to: Larisa Dzirlo, Third Department of Internal Medicine and Psychosomatics, St. Vincent Hospital, Vienna, Austria, E-mail: larisa.dzirlo@chello.at

Received: August 11, 2016; Accepted: August 31, 2016; Published: September 03,2016 
Dzirlo L (2016) Atypical recovery from stress induced cardiomyopathy in a patient with anorexia nervosa

3. Shin DG, Cho IJ, Shim CY, Ryu SK, Chang HJ, et al. (2015) Transient apical wall thickening in patients with stress cardiomyopathy: Prevalence, profile and impact on clinical course. Int J Cardiol 194: 87-92. [Crossref]
4. Osadchii OE (2007) Cardiac hypertrophy induced by sustained beta-adrenoreceptor activation: pathophysiological aspects. Heart Fail Rev 12: 66-86.[Crossref]

Copyright: ( 2016 Dzirlo L. This is an open-access article distributed under the terms of the Creative Commons Attribution License, which permits unrestricted use, distribution, and reproduction in any medium, provided the original author and source are credited. 